\title{
Paper-based standard addition assays $\uparrow$
}

Cite this: Anal. Methods, 2014, 6, 1296

\author{
Cory A. Chaplan, $\$$ Haydn T. Mitchell $\$$ and Andres W. Martinez*
}

Received 22nd January 2014

Accepted 27th January 2014

DOI: $10.1039 / c 4 a y 00205 a$

www.rsc.org/methods

Standard addition assays conducted on paper-based microfluidic devices are introduced as an alternative to external standards for calibrating quantitative tests. To demonstrate this technique, a colorimetric, paper-based, standard addition assay was optimized for the determination of glucose concentrations in the range of 0 to $5 \mathrm{mM}$. Comparable results were obtained from the assay via digital image colorimetry under three different lighting conditions.

In this communication, we introduce standard addition assays as an alternative to external standards for the quantitative detection of analytes on paper-based microfluidic devices (microPADs). Paper-based standard addition assays can be performed in a single sample addition step, and the technique is particularly appealing for quantifying the results of colorimetric assays since it does not require reproducible lighting conditions for digital image colorimetry. We demonstrated the technique by measuring the concentration of glucose in several solutions under three different lighting conditions.

The development of portable, user-friendly and quantitative diagnostic assays is an important part of efforts to improve healthcare in developing countries and remote settings. ${ }^{1}$ Porous membrane-based devices, such as dipsticks, lateral-flow devices and paper-based microfluidic devices, are among the most promising platforms for simple point-of-care diagnostics and typically support colorimetric assays. ${ }^{1-4}$ On their own, colorimetric assays provide only qualitative results, but in combination with a digital camera, colorimetric assays can provide quantitative results via digital image colorimetry (DIC). ${ }^{5-8}$

In DIC, the concentration of analytes is determined by taking a digital picture of a concluded colorimetric assay, reading the intensity of the color in the image, and then comparing the intensity to an external calibration curve. ${ }^{8}$ This technique is

Department of Chemistry \& Biochemistry, California Polytechnic State University, San Luis Obispo, CA 93401, USA. E-mail: awmartin@calpoly.edu

$\dagger$ Electronic supplementary information (ESI) available. See DOI: 10.1039/c4ay00205a

\$ These authors contributed equally to this project. appealing for the quantification of analytes in the field because it can be performed using a digital camera, ${ }^{6}$ it is compatible with porous-membrane-based diagnostic devices, ${ }^{8}$ and colorimetric assays already exist for a large number of analytes. ${ }^{9}$ The problem with DIC, in the context of point-of-care testing, is that the intensities of the colors in a digital image vary with the lighting conditions under which the image is obtained. ${ }^{\mathbf{1 0}}$ Therefore, if an assay is imaged under one set of lighting conditions in the field, and the color intensity is compared to an external calibration curve that was prepared in a laboratory under a different set of lighting conditions, then the results would not be accurate. , $^{\mathbf{1 0}}$

Three different approaches have been described for overcoming the lighting intensity problem associated with DIC: (i) controlling the lighting conditions in the field by using a portable scanner, a special light, or a light-box; ${ }^{\mathbf{4 1 1 - 1 3}}$ (ii) analyzing a series of external standard solutions in parallel with the unknown, and imaging all the results at the same time under the same lighting conditions; and (iii) imaging the device alongside a color calibration chart, and using the calibration chart and an associated algorithm to compensate for variations in lighting conditions. ${ }^{\mathbf{1 0}}$ The first two approaches are inconvenient for point-of-care diagnostics since they require additional equipment, reagents and time. The third approach was studied in detail by Murdock et al., and they concluded that color calibration charts would not allow for accurate calibration of colorimetric assays that produce varying intensities of the same color, and that a series of external standard solutions would most likely still need to be analyzed and imaged in parallel with the sample in order to account for variations in lighting conditions. ${ }^{\mathbf{1 4}}$ Ultimately, all three approaches rely on an external calibration curve and are not ideal for point-of-care colorimetric assays.

The method of standard additions provides an alternative to external standard calibration. Standard addition assays are used in quantitative analysis to overcome matrix effects that can affect the signal of an assay. ${ }^{15}$ In a traditional single-point standard addition assay, the signal of an unknown sample is 
measured, and then, the signal of the unknown plus a spike of analyte of known concentration is measured. From these data, one can calculate the concentration of the unknown sample without relying on an external calibration curve. ${ }^{16}$ Since standard addition assays do not rely on an external calibration curve, the results are not affected by variations in lighting conditions. While traditional standard addition assays are timeconsuming since several solutions must be prepared and tested for each sample to be analyzed, ${ }^{15}$ multiple standard addition assays can be performed simultaneously on a microPAD from a single sample-addition step. The reagents and the spike of analyte can both be added to the device during fabrication, stored on-chip and then re-suspended in the sample once the sample is added to the device (Fig. 1). ${ }^{17}$ This approach allows for spikes of analyte to be added to unknown samples automatically, without any additional input from the user and without diluting the sample to any significant extent.

We chose glucose as the analyte to demonstrate the technique for two principal reasons: (i) the measurement of glucose by DIC on paper-based devices using external calibration curves has been well characterized, ${ }^{8}$ and (ii) glucose can be dried in a channel of a paper-based microfluidic device and will dissolve in a sample solution as the solution wicks along the channel, ${ }^{17}$ which is a requirement for this particular form of paper-based standard addition assay. We optimized an enzymatic assay for glucose using 2,2'-azino-bis(3-ethylbenzothiazoline-6-sulfonic acid) diammonium salt (ABTS), which produced a blue-green colored product when glucose was present in the sample. ${ }^{\mathbf{1 9 , 2 0}}$ The standard addition assay was optimized to determine glucose concentrations in the range of 0 to $5 \mathrm{mM}$, since glucose levels in urine above $1.4 \mathrm{mM}$ can be indicative of disease. ${ }^{18}$

We fabricated paper-based microfluidic devices by wax printing with a central sample zone from which the sample was distributed through eight channels (spokes) into eight test zones (Fig. 1). ${ }^{21}$ The device was designed to run four replicates of sample $\left(S_{0}\right)$ and four replicates of sample spiked with a known concentration of glucose $\left(S_{1}\right)$. A reagent mixture containing $25 \mathrm{mM}$ ABTS, $75 \mathrm{kU} \mathrm{L}^{1}$ glucose oxidase, $250 \mathrm{kU} \mathrm{L}{ }^{1}$ horseradish peroxidase and $1 \mathrm{M}$ trehalose dissolved in 1 PBS

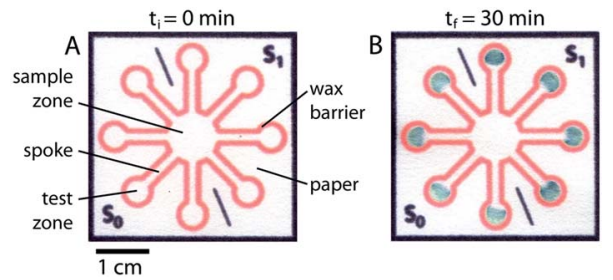

Fig. 1 Paper-based standard addition devices. (A) A device before addition of the sample. Reagents for the colorimetric glucose assay were dried in each test zone. A glucose spike solution was dried in the four spokes on the side of the device labeled $S_{1}$. (B) A device 30 minutes after adding a sample containing $2.5 \mathrm{mM}$ glucose to the sample zone. When the sample is added to the device, it wicks along the spokes dissolving any dried glucose and transporting it into the test zones. The intensity of the color in the $S_{1}$ test zones is always greater than that in the $S_{0}$ test zones because of the additional glucose from the spokes. was added to each test zone, and a $5.0 \mathrm{mM}$ glucose spike solution was added to the spokes of the $S_{1}$ sample zones. We then tested a series of glucose solutions prepared in 1 PBS by adding $35 \mu \mathrm{L}$ of the sample solution to the sample zone. No other action from the user was required, and the assays were complete after 30 minutes (please see the ESI for a discussion of the experimental design and additional experimental details $\dagger$ ).

To demonstrate the capability of the standard addition assay to provide quantitative results via DIC that were independent of lighting conditions, the devices were imaged under three different lighting conditions: (i) scanner with an LED light source, (ii) digital camera outdoors in sunlight and (iii) digital camera indoors under fluorescent lighting. The color intensities of the test zones in the red channel of the digital images were read using ImageJ.

Since the intensity of the color of paper-based colorimetric assays is usually not directly proportional to the concentration of analyte, ${ }^{8}$ a non-linear standard addition assay was developed. We chose a rectangular hyperbolic equation as the basis for the non-linear standard addition calculations because this equation described accurately the relationship between signal and concentration for the glucose assay (Fig. 2), and it allowed for the derivation of an equation relating the concentration of glucose to the signal from the assay. The signal from the assay $(S)$ can be described as:

$$
S=\frac{S_{\max } C}{K+C}
$$

where $S_{\max }$ is the maximum signal (color intensity) that can be achieved, $C$ is the concentration of analyte, and $K$ is a constant equal to the concentration of analyte at which half of the maximum signal is achieved. Rewriting eqn (1) in the context of a single-point standard addition assay yields two equations:

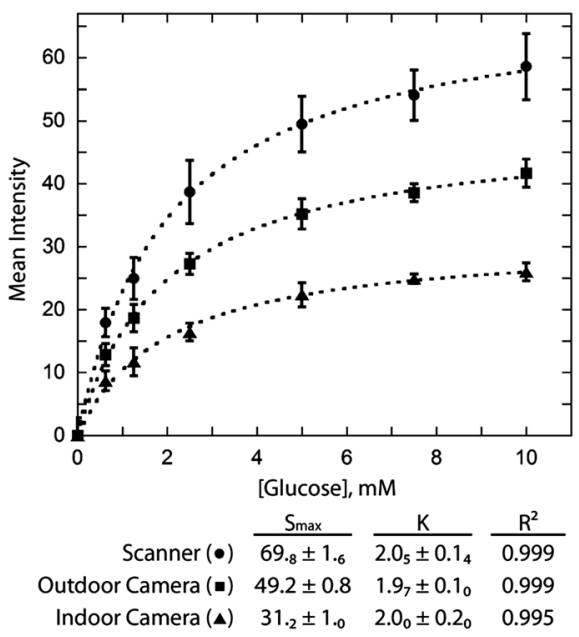

Fig. 2 External calibration curves for the glucose assay under three lighting conditions: scanner $(\mathbf{O})$, outdoor camera $(\mathbf{\square})$ and indoor camera ( $\mathbf{\Delta}$ ). Data points represent the mean of eight trials, and error bars represent one standard deviation from the mean. The curves were fit with eqn (1), and values for $S_{\max }, K$ and $R^{2}$ were obtained using KaleidaGraph®. 


$$
\begin{gathered}
S_{0}=\frac{S_{\max } C_{\mathrm{u}}}{K+C_{\mathrm{u}}} \\
S_{1}=\frac{S_{\max }\left(C_{\mathrm{u}}+C_{\mathrm{s}}\right)}{K+\left(C_{\mathrm{u}}+C_{\mathrm{s}}\right)}
\end{gathered}
$$

where $S_{0}$ is the signal from the unknown sample, $S_{1}$ is the signal from the unknown sample plus the spike of analyte, $C_{\mathrm{u}}$ is the concentration of the unknown, and $C_{\mathrm{s}}$ is the concentration of the spike. From eqn (2) and (3), we can solve for $C_{\mathrm{u}}$ in terms of $S_{0}, S_{1}, C_{\mathrm{s}}$, and either $S_{\max }$ or $K$. Since the intensities of color are known to vary with lighting conditions, we chose to solve for $C_{\mathrm{u}}$ in terms of $K$ under the hypothesis that the magnitude of $K$ would not vary with lighting conditions. The concentration of the unknown can thus be solved as:

$$
C_{\mathrm{u}}=\sqrt{\frac{\left(C_{\mathrm{s}}+K\right)^{2}}{4}+\frac{S_{0} C_{\mathrm{s}} K}{S_{1} \quad S_{0}}} \quad \frac{C_{\mathrm{s}}+K}{2}
$$

Eqn 4 was used to calculate the concentration of glucose in samples. The values for $S_{1}$ and $S_{0}$ came from the respective means of the color intensities of the four $S_{1}$ and $S_{0}$ test zones from each device. The value for $K$ was obtained from an external calibration curve that was fit with eqn (1) (Fig. 2), and the value for $C_{\mathrm{s}}$ was determined experimentally by testing known glucose solutions using devices with a spike of glucose in all eight spokes (Fig. 3).

The external calibration curves for the three different lighting conditions are shown in Fig. 2. All three curves have the same general shape, and fitting the curves with eqn (1) provided excellent fits, giving correlation values $\left(R^{2}\right)$ of $0.999,0.999$ and 0.995 for the scanner, outdoor camera and indoor camera respectively. As expected, the values of $S_{\max }$ varied significantly with lighting conditions. The value for $K$, on the other hand, was constant for all three lighting conditions with an average value of $2.0 \mathrm{mM}$. This result was critical to the success of this project and confirmed the initial hypothesis that the magnitude of $K$ would not be affected by lighting conditions.

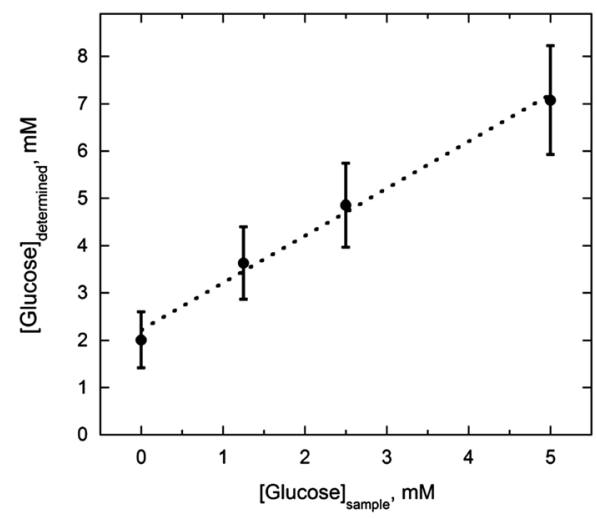

Fig. 3 Glucose spike calibration curve. Concentrations from each trial were determined using the scanner's external calibration curve. Each data point represents the mean of eight trials, and error bars represent one standard deviation from the mean. Linear regression analysis of the data gave a slope of $1.00 \quad 0.06$, an intercept of $2.2 \quad 0.2$ and an $R^{2}$ value of 0.992 for the best-fit line. The magnitude of the intercept represents the concentration of the spike $\left(C_{s}\right)$.
A second critical result was that the amount of glucose "spiked" into the sample from the spoke was constant for the range of glucose concentrations that were tested (Fig. 3). The average concentration of the spike $\left(C_{\mathrm{s}}\right)$ was $2.2 \mathrm{mM}$. This concentration was lower than the concentration of the $5.00 \mathrm{mM}$ glucose spike solution that was added to the spokes, which could be due to one or two reasons: (i) the glucose in the spoke did not dissolve completely in the sample, so less than half of the glucose added to the spoke actually reached the test zone, or (ii) the glucose dried in the spoke was diluted compared to its initial concentration. Ultimately, the source of the difference in concentrations is irrelevant to the results of the standard addition assay.

Using a value of $2.0 \mathrm{mM}$ for $\mathrm{K}$, a value of $2.2 \mathrm{mM}$ for $C_{\mathrm{s}}$ and the average values for $S_{1}$ and $S_{0}$ obtained from the scanned images for each device, the concentration of glucose in five different samples were determined using eqn (4) (Table 1). These results illustrate the accuracy and precision of the standard addition assay. While a large relative range was observed for the three trials of each sample, the mean value was always within one standard deviation of the true value. These results are typical of paper-based colorimetric assays, which tend to display a low level of precision, but relatively good accuracy when multiple trials are averaged to minimize the effects of indeterminate error. ${ }^{8}$ Using a 3D-microPAD, ${ }^{22,23}$ it should be possible to run dozens or even hundreds of replicates for $S_{0}$ and $S_{1}$ simultaneously on a single device, which should further improve the accuracy of the results. It should also be possible to perform multi-point standard addition assays by spiking the sample with different concentrations of analyte, which could also improve the accuracy of the results. The precision of the assays could likely be improved by using an automated liquid dispensing system to deposit the reagents on the devices.

The results from the three different lighting conditions are shown in Fig. 4 and Table 2. The results from the standard addition assays retain the same level of accuracy under different lighting conditions, while the accuracy of the results from the external calibration assays decreases significantly when the lighting conditions of the external standards do not match the lighting conditions of the samples (Fig. 4, Table S1†). A $t$-test confirmed there was no statistically significant difference between the results from the external calibration assay with controlled lighting conditions (scanner) and the standard

Table 1 Results from standard addition assays imaged with a scanner

\begin{tabular}{lcllll}
\hline Sample & $\begin{array}{l}\text { Trial 1 } \\
(\mathrm{mM})\end{array}$ & $\begin{array}{l}\text { Trial 2 } \\
(\mathrm{mM})\end{array}$ & $\begin{array}{l}\text { Trial 3 } \\
(\mathrm{mM})\end{array}$ & \multicolumn{2}{c}{$\begin{array}{l}\text { Mean } \\
\text { dev. }(\mathrm{mM})\end{array}$} \\
\hline $0 \mathrm{mM}$ & 0.03 & 0.02 & 0.06 & 0.02 & 0.04 \\
$1.25 \mathrm{mM}$ & 1.49 & 1.03 & 1.46 & 1.3 & 0.3 \\
$2.50 \mathrm{mM}$ & 2.34 & 2.63 & 2.12 & 2.4 & 0.3 \\
$5.00 \mathrm{mM}$ & 4.72 & 5.24 & 4.84 & 4.9 & 0.3 \\
Artificial urine $^{a}$ & 2.49 & 1.89 & 2.23 & 2.2 & 0.3 \\
Unknown $^{b}$ & 2.81 & 2.40 & 2.19 & 2.5 & 0.3
\end{tabular}

${ }^{a}$ Contained $2.5 \mathrm{mM}$ glucose and $5 \mathrm{mM}$ tartrazine, a yellow dye, to simulate the color of urine. ${ }^{b}$ Contained $2.5 \mathrm{mM}$ glucose and was analyzed in a blind experiment. 


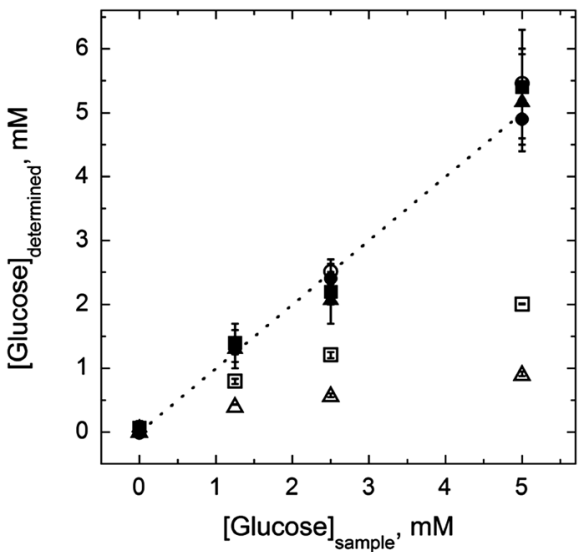

Fig. 4 Comparison of the standard addition assay results (solid shapes) to external calibration results (open shapes) for the three lighting conditions: scanner $(\bullet, \bigcirc)$, outdoor camera $(\boldsymbol{\square}, \square)$ and indoor camera $(\boldsymbol{\Lambda}, \Delta)$. The external calibration results for all three lighting conditions were calculated using the scanner's external calibration curve. Data points represent the mean of three trials, and error bars represent one standard deviation from the mean. The dashed line represents the desired result where the determined concentration equals the actual concentration of glucose in the sample.

Table 2 Results from the standard addition assays determined for the three lighting conditions. External calibration results for the scanner data are also shown for comparison. Results are all reported as the mean standard deviation for three trials

\begin{tabular}{|c|c|c|c|c|c|c|c|c|}
\hline \multirow{2}{*}{$\frac{\text { Sample }}{0 \mathrm{mM}}$} & \multicolumn{2}{|c|}{$\begin{array}{l}\text { Scanner } \\
(\mathrm{mM})\end{array}$} & \multicolumn{2}{|c|}{$\begin{array}{l}\text { Outdoor } \\
\text { camera }(\mathrm{mM})\end{array}$} & \multicolumn{2}{|c|}{$\begin{array}{l}\text { Indoor } \\
\text { camera }(\mathrm{mM})\end{array}$} & \multicolumn{2}{|c|}{$\begin{array}{l}\text { Scanner } \\
\text { ext. cal. }(\mathrm{mM})\end{array}$} \\
\hline & 0.02 & 0.04 & 0.07 & 0.03 & 0.10 & 0.07 & 0.02 & 0.03 \\
\hline $1.25 \mathrm{mM}$ & 1.3 & 0.3 & 1.4 & 0.3 & 1.3 & 0.1 & 1.31 & 0.09 \\
\hline $2.50 \mathrm{mM}$ & 2.4 & 0.3 & 2.1 & 0.2 & 2.1 & 0.4 & 2.5 & 0.1 \\
\hline $5.00 \mathrm{mM}$ & 4.9 & 0.3 & 5.4 & 0.9 & 5.2 & 0.8 & 5.4 & 0.5 \\
\hline Art. urine $\mathrm{e}^{a}$ & 2.2 & 0.3 & 2.4 & 0.5 & 2.2 & 0.3 & 2.58 & 0.07 \\
\hline Unknown $^{b}$ & 2.5 & 0.3 & 2.2 & 0.5 & 2.1 & 0.6 & 2.8 & 0.2 \\
\hline
\end{tabular}

${ }^{a}$ Contained $2.5 \mathrm{mM}$ glucose and $5 \mathrm{mM}$ tartrazine, a yellow dye, to simulate the color of urine. ${ }^{b}$ Contained $2.5 \mathrm{mM}$ glucose and was analyzed in a blind experiment.

addition assays under all three lighting conditions at the 95\% confidence level. The assay is most precise at lower concentrations of glucose where the differences between $S_{1}$ and $S_{0}$ are largest. At higher concentrations of glucose, as the difference between $S_{1}$ and $S_{0}$ decreases, the precision of the results tends to decrease. It should be possible to tune the range of concentrations of glucose that can be detected using this method by changing the concentration of glucose in the spike.

We demonstrated a paper-based, colorimetric, standard addition assay for glucose that is simple to perform and provides quantitative results by DIC that are independent of lighting conditions. These advantages are important in the context of point-of-care diagnostics and telemedicine, especially since lighting conditions in the field are difficult to control. In its present form, the paper-based standard addition assay can only be used for analytes that can be dried on a device, stored and then re-suspended in a sample, but the technique is not limited to colorimetric assays. Paper-based standard additions could be adapted to any type of paper-based assay including fluorescence assays, absorbance assays, electrochemical assays and chronometric assays. ${ }^{24-30}$ The quantitative results of these assays are not typically affected by ambient lighting conditions, but the method of standard additions could still be useful for overcoming matrix effects and for calibrating devices in the field without the need for a standard reference solution.

\section{Acknowledgements}

This project was supported by a Non-Tenured Faculty Grant from 3M, the Extramural Funding Initiative (EFI) at California Polytechnic State University, San Luis Obispo, and the California State University Program for Education and Research in Biotechnology (CSUPERB). We would like to thank Emily Fogle for help with fitting the calibration curves in Kaleidgraph.

\section{References}

1 C. D. Chin, V. Linder and S. K. Sia, Lab Chip, 2007, 7, 41-57. 2 P. von Lode, Clin. Biochem., 2005, 38, 591-606.

3 A. W. Martinez, S. T. Phillips, M. J. Butte and G. M. Whitesides, Angew. Chem., Int. Ed., 2007, 46, 13181320.

4 A. K. Yetisen, M. S. Akram and C. R. Lowe, Lab Chip, 2013, 13, 2210-2251.

5 W. Colin, E. Mark, M. Bonner and R. S. Pomeroy, Trends Anal. Chem., 1993, 12, 395-403.

6 L. Byrne, J. Barker, G. Pennarun-Thomas and D. Diamond, Trends Anal. Chem., 2000, 19, 517-522.

7 A. Lopez-Molinero, D. Liñan, D. Sipiera and R. Falcon, Microchem. J., 2010, 96, 380-385.

8 A. W. Martinez, S. T. Phillips, E. Carrilho, S. W. Thomas, H. Sindi and G. M. Whitesides, Anal. Chem., 2008, 80, 3699-3707.

9 E. Jungreis, in Spot Test Analysis: Clinical, Environmental, Forensic, and Geochemical Applications, 2nd edn, John Wiley \& Sons, Inc., New York, 1997.

10 L. Shen, J. A. Hagen and I. Papautsky, Lab Chip, 2012, 12, 4240-4243.

11 O. Mudanyali, S. Dimitrov, U. Sikora, S. Padmanabhan, I. Navruz and A. Ozcan, Lab Chip, 2012, 12, 2678-2686.

12 uChek, Biosense Technologies Pvt. Ltd, http://www.uchek.in. 13 D. Lee, B. G. Jeon, C. Ihm, J. Park and M. Y. Jung, Lab Chip, 2011, 11, 120-126.

14 R. C. Murdock, L. Shen, D. K. Griffin, N. Kelley-Loughnane, I. Paputsky and J. A. Hagen, Anal. Chem., 2013, DOI: 10.1021/ac403040a, just accepted manuscript.

15 D. Harvey, in Analytical Chemistry 2.0, published online at http://www.asdlib.org/onlineArticles/ecourseware/Welcome. htmlhttp://www.asdlib.org/onlineArticles/ecourseware/Wel come.html, 2008, pp. 161-167.

16 The relationship between signal and concentration must be known, and standard addition assays are typically 
performed for assays with a linear relationship between signal and concentration.

17 K. C. Schilling, D. Jauregui and A. W. Martinez, Lab Chip, 2013, 13, 628-631.

18 J. K. Davidson, in Clinical Diabetes Mellitus: A ProblemOriented Approach, 3rd edn, Thieme, New York, 2000, p. 444.

19 D. R. Nelson and A. K. Huggins, Anal. Biochem., 1974, 59, 46-53.

20 R. C. Bateman and J. A. Evans, J. Chem. Educ., 1995, 72, A240A241.

21 E. Carrilho, A. W. Martinez and G. M. Whitesides, Anal. Chem., 2009, 81, 7091-7095.

22 A. W. Martinez, S. T. Phillips and G. M. Whitesides, Proc. Natl. Acad. Sci. U. S. A., 2008, 105, 19606-19611.

23 G. G. Lewis, M. J. DiTucci, M. S. Baker and S. T. Phillips, Lab Chip, 2012, 12, 2630-2633.
24 E. Carrilho, S. T. Phillips, S. J. Vella, A. W. Martinez and G. M. Whitesides, Anal. Chem., 2009, 81, 5990-5998.

25 N. K. Thom, K. Yeung, M. B. Pillion and S. T. Phillips, Lab Chip, 2012, 12, 1768-1770.

26 A. K. Ellerbee, S. T. Phillips, A. C. Siegel, K. A. Mirica, A. W. Martinez, P. Striehl, N. Jain, M. Prentiss and G. M. Whitesides, Anal. Chem., 2009, 81, 8447-8452.

27 W. Dungchai, O. Chailapakul and C. S. Henry, Anal. Chem., 2009, 81, 5821-5826.

28 Z. Nie, C. A. Nijhuis, J. Gong, X. Chen, A. Kumachev, A. W. Martinez, M. Narovlyansky and G. M. Whitesides, Lab Chip, 2010, 10, 477-483.

29 J. P. Metters, S. M. Houssein, D. K. Kampouris and C. E. Banks, Anal. Methods, 2013, 5, 103-110.

30 G. G. Lewis, M. J. DiTucci and S. T. Phillips, Angew. Chem., Int. Ed., 2012, 51, 12707-12710. 